\title{
Dyslipidemia in Newly Diagnosed Diabetic Patients With or Without Micro Albuminuria
}

Naimatullah Khan, Javed Iqbal Khan, Tariq Mehmood, Aqeel Peter

\section{ABSTRACT}

Objective: To assess the dyslipidemia in newly diagnosed diabetic patients and compare it with or without micro albuminuria.

Study Design and Setting: Cross-sectional study at Department of Medicine, Military Hospital Rawalpindi from 01 March 2015 to 31 August 2015.

Methodology: A total of 150 patients who fulfilled the inclusion criteria were included after taking informed consent and approval from hospital ethical committee. Blood sample was obtained from each patient and samples were sent to hospital laboratory and reports were assessed by pathologist. Presence or absence of microalbuminuria, trigiyceridemia (200-499 $\mathrm{mg} / \mathrm{dl})$, raised LDL-C $(>160 \mathrm{mg} / \mathrm{dl})$ and decreased HDL-C $(<40 \mathrm{mg} / \mathrm{dl})$ were noted by researcher himself on a pre-designed performa. The collected data was analyzed statistically by using SPSS version 12.Quantitative variables like age, TG, LDL HDL levels were presented in form of mean \pm S.D. Qualitative variables like gender, diabetes mellitus, micro albuminuria and dyslipidemia were presented as frequencies and percentages. Chi-square test was applied to find association between dyslipidemia and micro albuminuria. $\mathrm{P}$ value $=0.05$ was considered as statistically significant.

Results: Mean \pm SD of age was $47.97 \pm 7.08$ years. Out of 150 patients $60 \%$ were male and $40 \%$ were female. Dyslipidemia was found in $42 \%$ patients. Highly significant association was found between dyslipidemia and micro albuminuria, i.e. $(\mathrm{P}=0.0001)$.

Conclusion: The frequency of dyslipidemia among newly diagnosed diabetic patients with micro albuminuria was significantly higher than in subjects without micro albuminuria.

Keywords: Dyslipidemia, Micro albuminuria, Newly diagnosed diabetic patients.

\section{INTRODUCTION:}

Over the last two decades Diabetes Mellitus (DM) has emerged as one of the challenging epidemics worldwide. ${ }^{1}$ Initially DM was considered to be prevalent among developing countries, disease of privileged and senior citizens. It has now become common in developing nation irrespective of age, culture and socioeconomic status. South Asia owing to its demography, lifestyle and dietary habits of its population is emerging as epicenter of this global epidemic. ${ }^{1} \mathrm{DM}$ is affecting more than 150 million people worldwide. It is estimated that the number of people with DM is expected to rise to 552 million by 2030 , with a $69 \%$ increase from 2010 to 2030 for developing countries, compared to $20 \%$

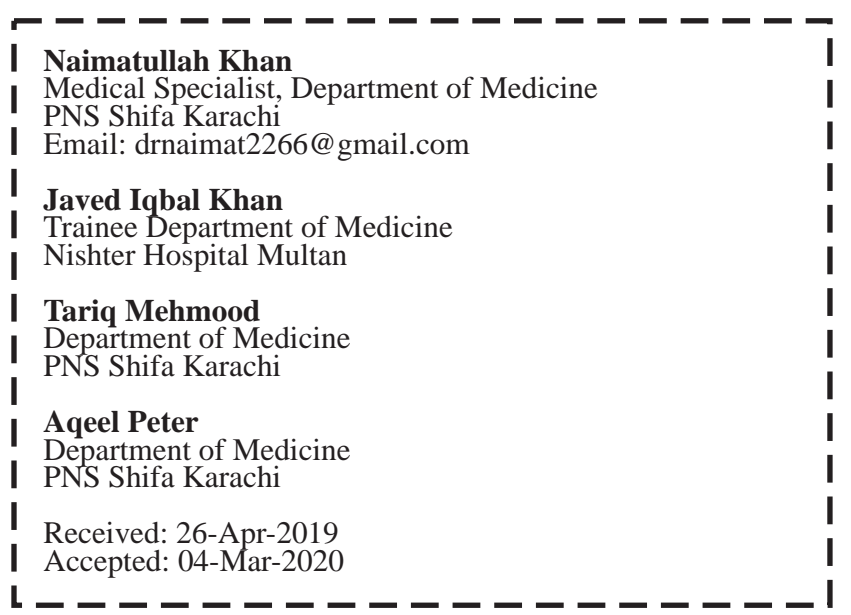

for developed countries. ${ }^{2,3}$ In Pakistan DM is rising at startling rate. According to World Health Organization report, about 12.9 million individuals are suffering from DM and this count accounts for about $10 \%$ of total population. ${ }^{4}$ According to a survey Pakistan stood among top ten countries in the world with most cases of type 2 Diabetes Mellitus (T2DM). ${ }^{5}$

DM is known from ancient times. However, Aulus Cornelius Celsus (30 BC-50 AD) was the first one to describe the symptoms of this disease ${ }^{6}$ Over time the disease has been extensively studied with regards to its genetics, pathophysiology and treatment options. Today it is considered a complex heterogeneous entity requiring multidisciplinary approach. It progresses slowly over time leading to secondary complications or some time may present suddenly as coma. It is a multifactorial disease usually resulting from interplay between genetic and environmental factors. Genome-Wide Association Studies has shown that individuals with certain genetic variations are more likely to develop this disease ${ }^{7}$. Main pathophysiology leading to this metabolic syndrome is hyperglycemia resulting from either decreased insulin secretion or impaired insulin action on target tissues. This hyperglycemia along with poor circulation in Diabetes Mellitus can harm various organs including kidneys and heart. ${ }^{8}$ Presence of high levels of protein (albumin) in the urine is an early sign of kidney damage. Micro albuminuria is defined as albumin excretion rate of $30-300 \mathrm{mg} / \mathrm{day}$. Frequency of micro albuminuria in newly diagnosed diabetics 
was $50 \% .{ }^{9}$ Dyslipidemia is characterized by the elevation of plasma total cholesterol (TC), triglycerides (TG), reduced high- density lipoprotein cholesterol (HDL-C), and increased low-density lipoprotein cholesterol (LDL-C). It contributes significantly to the excess risk of cardiovascular disease. ${ }^{10}$ The prevalence of hypercholesterolemia, hypertriglyceridemia, low HDL cholesterol and high LDL cholesterol in subjects with newly diagnosed Diabetes Mellitus are $67.7 \%, 54.9 \%, 36.8 \%$ and $91.7 \%$ respectively. ${ }^{11}$ Results of some studies show association between micro albuminuria and dyslipidemia. In one study, in diabetic patients with and without albuminuria, hypertriglyceridemia was 93.7 vs. $67.3 \%$, decreased HDL-C was $91.3 \%$ vs. $81.4 \%$ and raised LDL-C was $93.2 \%$ vs. $69.5 \%$. However there is a contradiction, as albuminuria was found to be associated with low HDL-C not with TC, TG, and LDL-C. But some researchers have found significant differences in TC, TG and HDL-C between those with or without albuminuria respectively ${ }^{12}$. Despite all this there is increasing evidence of relationship between dyslipidemias and albuminuria. Several mechanism have been hypothesized to explain this association. According to one TG rich lipoprotein activates transforming growth factor â pathway (TGF- â) which promotes production of reactive oxygen species (ROS). ROS can cause glomerular damage ${ }^{13}$.

Although the relationship between dyslipidemias, renal disease and DM has been investigated extensively. There is still a need to study these associations in our population which will not only improve our health care delivery system and protocols but also improve patients holistic health. Keeping this in mind we conducted this study at our clinical setup so as to improve our clinical practice and bring it up to international benchmarks. Therefore the aim of this study was to determine the frequency of dyslipidemia in newly diagnosed diabetic patients and compare it with or without micro albuminuria.

As diabetic patients with micro albuminuria are at more risk of developing cardiac and renal problems but often remain undiagnosed for presence of dyslipidemia. This study would be helpful to manage the cases accordingly and to prevent mortalities and morbidities.

\section{METHODOLOGY:}

After taking approval from Hospital ethical committee, I50 patients (sample size was calculated using WHO sample size calculator) who fulfilled the inclusion criteria, i.e. patients having age range 20-70 years of either gender and patients with newly diagnosed type 2 diabetes mellitus (diagnosed within 1 year), were enrolled in the study from OPD. Patients with history of smoking, pregnancy, long standing diabetes, elevated creatinine, overt proteinuria, patients on lipid lowering drugs, ACE inhibitors or angiotensin receptor blockers, hypertensive patients, patients with non-diabetic renal disease and heart failure, were excluded from the study. The purpose of the study was explained to each patient and informed consent was obtained. All basic demographic information of each patient (name, gender, address and contact number) was noted. Blood samples were obtained from each patient and samples were sent to hospital laboratory and reports were assessed by pathologist. Presence or absence of microalbummuria, triglyceridemia (200-499 mg/dl), raised LDL-C(>160 mg/dl) and decreased HDL-C $(<40 \mathrm{mg} / \mathrm{dl})$ were noted. Deranged lipid profile was assessed by spectrophotometric absorption.

The collected data was analyzed statistically by using SPSS version 12.Quantitative variables like age, TG, LDL HDL levels were presented in form of mean \pm S.D. Qualitative variables like gender, diabetes mellitus, micro albuminuria and dyslipidemia were presented as frequency and percentage. Chi-square test was applied to find association between dyslipidemia and micro albuminuria. Effect modifiers were controlled through stratification of age, gender, duration and type of diabetes mellitus to see the effect of these on outcome variables. Post stratification, Chi-square was applied considering $\mathrm{P}=0.05$ as significant.

\section{RESULTS:}

In this study 150 newly diagnosed type 2 diabetic patients were included to assess the dyslipidemia with and without micro albuminuria and results were analyzed as follows:

Mean \pm SD of age was $47.97 \pm 7.08$ years. Mean \pm SD of LDL, TG and HDL were $113.67 \pm 19.50,160.68 \pm 19.30$ and $41.32 \pm 7.77 \mathrm{mg} / \mathrm{dl}$ respectively. Out of 150 patients $90(60 \%)$ were male and $60(40 \%)$ were female. Dyslipidemia was found in $63(42 \%)$ patients. Micro albuminuria was found in $18(12 \%)$ patients. In comparison of dyslipidemia with and without micro albuminuria, $12 \%$ patients had dyslipedimia with micro albuminuria and highly significant association was found $(\mathrm{P}=0.0001)$.

Stratification of dyslipidemia with respect to age, gender, type and duration of diabetes mellitus, LDL, HDL and TG was done, as shown in Table 1 while comparison of dyslipidemias with microalbuminuria is shown in Table 2 .

\section{DISCUSSION:}

The prevalence of Type II DM is on the increase according to age in spite of awareness programs that have been done in high risk population about DM. The proportion of Type II DM is higher than that of Type I DM and the difference is almost doubled ${ }^{14}$. The clinical characteristics which consist of BMI, waist circumference, total cholesterol level, triglyceride level, HDL cholesterol level and LDL cholesterol level among subjects with newly diagnosed DM are similar to those of subjects with diagnosed $\mathrm{DM}^{15}$. The most common pattern of dyslipidemia in DM are hypertriglyceridemia and low HDL cholesterol level ${ }^{16}$. In this study the proportion of dyslipidemia is significantly higher in patients with micro albuminuria as compared to that without micro albuminuria. 
Naimatullah Khan, Javed Iqbal Khan, Tariq Mehmood, Aqeel Peter

Table 1. Stratification of Dyslipidemia according toAge groups, Gender, Type and Duration of DM,LDL, HDL and TG

\begin{tabular}{|c|c|c|c|c|c|}
\hline \multirow{2}{*}{$\begin{array}{c}\text { Clinicopathological } \\
\text { variable }\end{array}$} & \multirow{2}{*}{$\begin{array}{c}\text { Cases } \\
(\mathrm{n}=150)\end{array}$} & \multirow{2}{*}{$\begin{array}{c}\text { Percentage } \\
(\%)\end{array}$} & \multicolumn{2}{|c|}{ Dyslipidemia } & \multirow{2}{*}{$\begin{array}{c}P \\
\text { Value }\end{array}$} \\
\hline & & & Yes & No & \\
\hline Age Groups (years) & & & & & \\
\hline $27-47$ & 68 & 45 & 11 & 57 & 0.0001 \\
\hline$>47$ & 82 & 55 & 52 & 30 & \\
\hline Gender & & & & & \\
\hline Male & 90 & 60 & 38 & 52 & 0.0001 \\
\hline Female & 60 & 40 & 25 & 35 & \\
\hline $\begin{array}{l}\text { Type of DM } \\
\text { Type II }\end{array}$ & 150 & 100 & 63 & 87 & NA \\
\hline Duration (months) & & & & & \\
\hline $0-6$ & 68 & 45 & 30 & 38 & 0.632 \\
\hline $7-12$ & 82 & 55 & 33 & 49 & \\
\hline LDL (mg/dl) & & & & & \\
\hline $89-113$ & 87 & 58 & 01 & 86 & 0.0001 \\
\hline$>113$ & 63 & 42 & 62 & 1 & \\
\hline HDL (mg/dl) & & & & & \\
\hline $25-41$ & 77 & 51 & 61 & 16 & 0.0001 \\
\hline$>41$ & 73 & 49 & 02 & 71 & \\
\hline TG (mg/dl) & & & & & \\
\hline $134-160$ & 93 & 62 & 06 & 87 & 0.0001 \\
\hline$>160$ & 57 & 38 & 57 & 00 & \\
\hline
\end{tabular}

Table 2. Comparison of Dyslipidemia With Micro Albumunria

\begin{tabular}{|c|c|c|c|}
\hline \multirow{2}{*}{ DYSLIPIDEMIA } & \multicolumn{2}{|c|}{ MICRO ALBUMINURIA } & \multirow{2}{*}{ P-VALUE } \\
\cline { 2 - 3 } & POSITIVE & NEGATIVE & \\
\hline YES & 15 & 48 & \multirow{2}{*}{0.0001} \\
\hline NO & 3 & 84 & \\
\hline
\end{tabular}

The mean LDL, HDL and TG levels among subjects with newly diagnosed DM with micro albuminuria is significantly different from that in those individuals who do not have micro albuminuria. Unfortunately, this study did not perform qualitative changes in LDL cholesterol, which in particular, theoretically the subjects with DM tend to have higher proportion of small dense LDL particles ${ }^{17}$. The mean \pm SD of LDL, TG and HDL was $113.67 \pm 19.50,160.68 \pm 19.30$ and $41.32 \pm 7.77$ respectively. As compared with the study of Goff DC et $\mathrm{al}^{18}$ who reported mean \pm SD of LDL,TG and HDL as $125.84 \pm 13.56,165.28 \pm 17.48$ and $44.69 \pm 9.34$ respectively, which is comparable with our study. In this study, the proportion of dyslipidemia is higher in male population. In our population the largest proportion is in the range of 30-40 and 40-50 years old and more than half of them have central obesity. The prevalence of hypertension, obesity and central obesity are significantly higher among subjects with dyslipidemia and Type II DM than those with only dyslipidemia. The occurrence of both dyslipidemia and newly diagnosed DM will increase CVD risk components several folds and most of those subjects do not realize the risks ${ }^{19,20}$. In our study, majority of patients had age group > 47 years $(55 \%)$ followed by age group $27-47$ years $(45 \%)$. This finding supported by the study conducted by Haffner
$\mathrm{SM}^{21}$ and Bays HE et $\mathrm{al}^{22}$, which shows that the commonest age of newly diagnosed diabetes between 35 to 70 years with mean age of 50 years. From this data, it can be concluded that aggressive screening and education among high risk population and in general as well are still needed. Since the newly diagnosed DM occurs two fold in the fourth and fifth decade, it is better to start screening population in the third decade and maybe in the second decade among high risk population $^{23}$. Unfortunately, this study did not collect history of the family, so the importance of genetic factor cannot be concluded. Among subjects without normal micro albuminuria, there is significant relationship between central obesity and all types of dyslipidemia. But, among subjects with newly diagnosed diabetes mellitus, the relationship between central obesity and dyslipidemia are not significant anymore. Thus, it may show that prevention should be started before glucose intolerance emerges. The occurrence of dyslipidemia among subjects with glucose intolerance will double the risk of $\mathrm{CVD}^{24}$. Screening among high risk population is the best way to detect metabolic changes prior to the occurrence of metabolic diseases. Besides that, earlier life style modification among general population may postpone or prevent CVD especially in high risk population ${ }^{25}$. Strength of the study was use of consecutive sampling best suited for our study design and sample selection, as our inclusion and exclusion criteria were stringent. The use of objective definitions for predictor and outcome variable also minimizes the source of bias in our study. The main limitations of our study were use of a weak study design that is cross-sectional analysis. Also limited outcomes selected in our study affects the worth of our study. There were many variables and factors that have association with our predictor and outcome variables that could have been included in our study. The use of non-probability sampling also limits generalizability; however we had a small number of patients and no follow up. This study was hospital-based study; hence the figure does not reflect true frequency and severity of the disease. Moreover, the study was conducted in one unit in single hospital which further confined its generalization. It is important for healthcare providers and policy makers to plan preventive strategies for micro albuminuria spread as well as diabetes prevention programs among high risk population. Effective preventive measures are the need of the hour and Pakistan may be considered as one of the areas of highest diabetes prevalence around the globe.

\section{CONCLUSION:}

The characteristics of newly diagnosed diabetes mellitus were similar to diagnosed diabetes mellitus. The frequency of dyslipidemia among newly diagnosed diabetic patients with micro albuminuria was significantly higher than that in subjects without micro albuminuria. 


\section{REFERENCES:}

1. Herath H, Weerasinghe N, Dias H, Weerarathna T. Knowledge, attitude and practice related to diabetes mellitus among the general public in Galle district in Southern Sri Lanka: a pilot study. BMC Public Health. 2017;17(1).

2. Sami W, Ansari T, Butt NS, Ab Hamid MR. Effect of diet on type 2 diabetes mellitus: A review. International journal of health sciences. 2017;11(2):65.

3. Previti E, Salinari S, Bertuzzi A, Capristo E, Bornstein S, Mingrone G. Glycemic control after metabolic surgery: a Granger causality and graph analysis. American Journal of Physiology - Endocrinology And Metabolism. 2017;:ajpendo. 00042.2017.

4. World Health Organization. Global report on diabetes [Internet]. Geneva: World Health Organization; 2016. Available from: http://www.who.int/diabetes/global-report/en/

5. Akhtar S, Khan Z, Rafiq M, Khan A. Prevalence of Type II diabetes in District Dir Lower in Pakistan. Pakistan Journal of Medical Sciences. 2016;32(3).

6. Abdel-Gayoum AG. The effect of glycemic control in type 2 diabetic patients with diabetes-related dyslipidemia. Saudi Med J. 2004;25:207-11.

7. Prasad R, Groop L. Genetics of Type 2 Diabetes-Pitfalls and Possibilities. Genes. 2015;6(4):87-123.

8. Cantley J, Ashcroft F. Q\&A: insulin secretion and type 2 diabetes: why do â-cells fail?. BMC Biology. 2015;13(1).

9. Afsar B, Elsurer R. The independent relationship between creatinine clearance, micro albuminuria and circadian blood pressure levels in newly diagnosed essential hypertensive and type 2 diabetic patients. J Diabetes Complications. 2012;26:5315.

10. Adar T, Lysy J. Pseudodyslipidemia: are we over-treating dyslipidemia in diabetic patients with undiagnosed gastroparesis? Endocrine. 2014;45:26-7.

11. Agaba EI, Agaba PA, Puepet FH. Prevalence of micro albuminuria in newly diagnosed type 2 diabetic patients in Jos Nigeria. Afr J Med Med Sci. 2004;33:19-22.

12. Chen SC, Tseng CH. Dyslipidemia, kidney disease, and cardiovascular disease in diabetic patients. Rev Diabet Stud. 2013;10:88-100.

13. Chen SC, Tseng CH. Dyslipidemia, kidney disease, and cardiovascular disease in diabetic patients. The review of diabetic studies: RDS. 2013;10(2-3):88.

14. Harris MI, Eastman RC. Early detection of undiagnosed diabetes mellitus: a US perspective. Diabetes/metabolism research and reviews. 2000;16:230-6.
15. Huang JW, Lien YC, Yang CY, Liu KL, Wu CF, Yen CJ, et al. Osteoprotegerin, inflammation and dyslipidemia are associated with abdominal aortic calcification in non-diabetic patients on peritoneal dialysis. NutrMetabCardiovasc Dis. 2014;24:236-42.

16. Jisieike-Onuigbo NN, Unuigbe EI, Kalu OA, Oguejiofor CO, Onuigbo PC. Prevalence of dyslipidemia among adult diabetic patients with overt diabetic nephropathy in Anambra state south-east Nigeria. Niger J ClinPract. 2011;14:171-5.

17. PascualFuster V, Ruiz Olivar E, Pinto Sala X. [Patient's care and management of dyslipidemia in type 2 diabetic patients in the clinical practice in Spain: The LIPEDIA study]. ClinInvestigArterioscler. 2015;27:45-56.

18. Goff DC, Bertoni AG, Kramer H, Bonds D, Blumenthal RS, Tsai MY, et al. Dyslipidemia prevalence, treatment, and control in the multi-ethnic study of atherosclerosis (MESA) gender, ethnicity, and coronary artery calcium. Circulation. 2006;113:647-56.

19. Xu Y, Bao Q, He B, Pan Y, Zhang R, Mao X, et al. Association of angiotensin I converting enzyme, angiotensin II type 1 receptor and angiotensin I converting enzyme 2 gene polymorphisms with the dyslipidemia in type 2 diabetic patients of Chinese Han origin. J Endocrinol Invest. 2012;35:378-83

20. Yadav D, Mishra M, Tiwari A, Bisen PS, Goswamy HM, Prasad GB. Prevalence of dyslipidemia and hypertension in Indian type 2 diabetic patients with metabolic syndrome and its clinical significance. Osong Public Health Res Perspect. 2014;5:169-75.

21. Haffner SM. American diabetes association. Dyslipidemia management in adults with diabetes. 2004.

22. Bays HE, Chapman R, Grandy S. The relationship of body mass index to diabetes mellitus, hypertension and dyslipidaemia: comparison of data from two national surveys. International journal of clinical practice. 2007;61:737-47.

23. Zimmet PZ, Alberti G. The Metabolic Syndrome: Perhaps an Etiologic Mystery but Far From a Myth-Where Does the International Diabetes Federation Stand? Medscape Diabetes \& Endocrinology. 2005;8.

24. Ormazabal V, Nair S, Elfeky O, Aguayo C, Salomon C, Zuñiga FA. Association between insulin resistance and the development of cardiovascular disease. Cardiovascular diabetology. 2018;17(1):122.

25. Nielsen JB, Leppin A, e Gyrd-Hansen D, Jarbøl DE, Søndergaard J, Larsen PV. Barriers to lifestyle changes for prevention of cardiovascular disease-a survey among 40-60year old Danes. BMC cardiovascular disorders. 2017;17(1):245 